The Astrophysical Journal, 654:570-579, 2007 January 1

(C) 2007. The American Astronomical Society. All rights reserved. Printed in U.S.A.

\title{
DISCOVERY OF TWO T DWARF COMPANIONS WITH THE SPITZER SPACE TELESCOPE
}

\author{
K. L. Luhman, ${ }^{1}$ B. M. Patten, ${ }^{2}$ M. Marengo, ${ }^{2}$ M. T. Schuster,${ }^{2,3}$ J. L. Hora, ${ }^{2}$ R. G. Ellis, ${ }^{2}$ J. R. Stauffer, ${ }^{4}$ \\ S. M. Sonnett, ${ }^{2,5}$ E. Winston, ${ }^{2,6}$ R. A. Gutermuth ${ }^{2,6}$ S. T. Megeath, ${ }^{7}$ D. E. Backman, ${ }^{8}$ T. J. Henry, ${ }^{9}$ \\ M. W. Werner, ${ }^{10}$ and G. G. FAZIO ${ }^{2}$ \\ Received 2006 July 22; accepted 2006 September 8
}

\begin{abstract}
We report the discovery of T dwarf companions to the nearby stars HN Peg (G0 V, $18.4 \mathrm{pc}, \tau \sim 0.3 \mathrm{Gyr})$ and HD 3651 ( K0 V, $11.1 \mathrm{pc}, \tau \sim 7 \mathrm{Gyr}$ ). During an ongoing survey of $5^{\prime} \times 5^{\prime}$ fields surrounding stars in the solar neighborhood with the Infrared Array Camera aboard the Spitzer Space Telescope, we identified these companions as candidate T dwarfs based on their mid-infrared colors. Using near-infrared spectra obtained with SpeX at the NASA Infrared Telescope Facility, we confirm the presence of methane absorption that characterizes T dwarfs and measure spectral types of $\mathrm{T} 2.5 \pm 0.5$ and T7.5 \pm 0.5 for HN Peg B and HD 3651B, respectively. By comparing our Spitzer data to images from the Two Micron All Sky Survey obtained several years earlier, we find that the proper motions of HN Peg B and HD 3651B are consistent with those of the primaries, confirming their companionship. A comparison of their luminosities to the values predicted by theoretical evolutionary models implies masses of $0.021 \pm 0.009$ and $0.051 \pm 0.014 M_{\odot}$ for HN Peg B and HD 3651B, respectively. In addition, the models imply an effective temperature for HN Peg B that is significantly lower than the values derived for other T dwarfs at similar spectral types, which is the same behavior reported by Metchev \& Hillenbrand for the young late L dwarf HD 203030B. Thus, the temperature of the L/T transition appears to depend on surface gravity. Meanwhile, HD 3651B is the first substellar companion directly imaged around a star that is known to harbor a close-in planet from radial velocity surveys. The discovery of this companion supports the notion that the high eccentricities of close-in planets like that near HD 3651 may be the result of perturbations by low-mass companions at wide separations.
\end{abstract}

Subject headings: binaries: visual — infrared: stars — stars: evolution — stars: fundamental parameters — stars: low-mass, brown dwarfs

\section{INTRODUCTION}

Direct-imaging surveys for resolved substellar companions are vital for studies of brown dwarfs and planets. These surveys provide measurements of the frequency of companions as a function of various physical properties, such as primary mass, companion mass, separation, and age, which in turn can be used to constrain the mechanisms by which substellar companions form. In addition, compared to their isolated counterparts, each newly uncovered companion represents a superior laboratory for studying the atmospheres and evolution of substellar objects because the age, distance, and metallicity of the primary, and hence the companion, are relatively easy to measure.

A variety of criteria can be adopted for selecting primaries to search for companions. Stars for which planets have been discovered through radial velocity monitoring and young nearby stars are particularly attractive categories to consider. Several

\footnotetext{
1 Department of Astronomy and Astrophysics, The Pennsylvania State University, University Park, PA; kluhman@astro.psu.edu.

2 Harvard-Smithsonian Center for Astrophysics, Cambridge, MA. $\mathrm{MN}$.

School of Physics and Astronomy, University of Minnesota, Minneapolis,

${ }_{5}^{4}$ Spitzer Science Center, California Institute of Technology, Pasadena, CA.

5 Institute for Astronomy, University of Hawaii at Manoa, Honolulu, HI.

6 Visiting Astronomer at the Infrared Telescope Facility, which is operated by the University of Hawaii under Cooperative Agreement NCC 5-538 with the National Aeronautics and Space Administration, Office of Space Science, Planetary Astronomy Program.

7 Department of Physics and Astronomy, University of Toledo, Toledo, $\mathrm{OH}$.

8 SOFIA/SETI Institute, Mountain View, CA.

9 Department of Physics and Astronomy, Georgia State University, Atlanta, GA.

io Jet Propulsion Laboratory, Pasadena, CA.
}

direct-imaging surveys have focused on known extrasolar planetary systems (Luhman \& Jayawardhana 2002; Patience et al. 2002; Mugrauer et al. 2005; Chauvin et al. 2006), producing one companion that was initially described as a brown dwarf (Els et al. 2001 ) but was later shown to be a white dwarf (Mugrauer \& Neuhäuser 2005; Lagrange et al. 2006). Similar observations have been performed toward young stars and brown dwarfs within 100 pc of the Sun ( $\tau \sim 10-400$ Myr; Chauvin et al. 2003; Neuhäuser et al. 2000a; McCarthy \& Zuckerman 2004; Lowrance et al. 2005), resulting in the discovery of several substellar companions that include G196-3B (Rebolo et al. 1998), TWA 5B (Lowrance et al. 1999; Neuhäuser et al. 2000b), HR 7329B (Lowrance et al. 2000; Guenther et al. 2001), AB Pic B (Chauvin et al. 2005b), HD 49197B (Metchev \& Hillenbrand 2004), 2MASS 1207-3932B (Chauvin et al. 2004, 2005a), and HD 203030B (Metchev \& Hillenbrand 2006). The spectral types of these companions range from late $\mathrm{M}$ through $\mathrm{L}$ but do not extend into the cooler $\mathrm{T}$ types. The only known T dwarf companions to stars consist of Gl 229B (Nakajima et al. 1995; Oppenheimer et al. 1995), Gl 570D (Burgasser et al. 2000), Gl 337D (Wilson et al. 2001; Burgasser et al. 2005), $\epsilon$ Ind B and C (Scholz et al. 2003; McCaughrean et al. 2004), and SCR 1845B (Biller et al. 2006), all of which orbit nearby stars that are older than $\sim 1$ Gyr and have no known planets.

Most resolved substellar companions have been identified through deep, high-resolution imaging at small separations ( $\rho \lesssim 10^{\prime \prime}$ ) with adaptive optics or the Hubble Space Telescope, or through shallow imaging at arbitrarily large separations with the Two Micron All Sky Survey (2MASS) or the Sloan Digital Sky Survey (SDSS). Because of its wide field of view $\left(\rho=2.5^{\prime}\right)$ and unprecedented sensitivity at mid-infrared (mid-IR) wavelengths, the Infrared Array Camera (IRAC; Fazio et al. 2004) on 

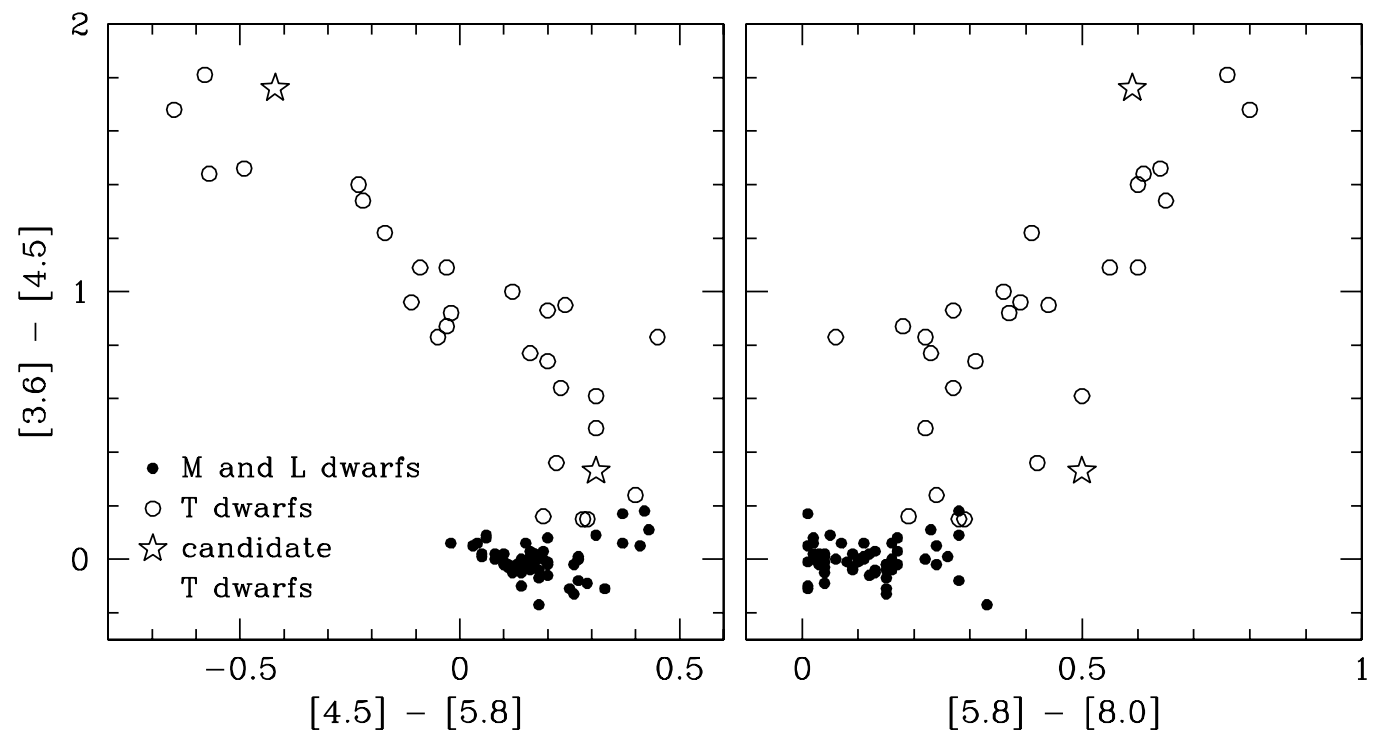

FIG. 1.-IRAC color-color diagrams for cool field dwarfs (open and filled circles; Patten et al. 2006) and two candidate companions of HN Peg (lower stars) and HD 3651 (upper stars). The colors of these candidates are indicative of T dwarfs.

board the Spitzer Space Telescope (Werner et al. 2004) complements these previous search methods by providing the best available sensitivity to low-mass companions at separations of $\rho \sim 10^{\prime \prime}-100^{\prime \prime}(r \sim 100-1000 \mathrm{AU})$ from nearby stars. To take advantage of these capabilities, we have obtained IRAC images of nearby young stars and planet-bearing stars identified in radial velocity surveys. In this paper, we report the discovery of two T dwarf companions to stars in these samples, HN Peg $(d=18.4 \mathrm{pc}, \mathrm{G} 0 \mathrm{~V})$ and HD $3651(d=11.1 \mathrm{pc}, \mathrm{K} 0 \mathrm{~V}) .{ }^{11}$ The former is a young solar analog (Gaidos 1998), while the latter has a sub-Saturn planetary companion at $0.3 \mathrm{AU}$ (Fischer et al. 2003; Fischer \& Valenti 2005). We begin by describing the IRAC images that were used to identify the $T$ dwarf companions to these stars ( $(2.1)$ and follow-up near-IR photometry and spectroscopy $(\S 2.2)$. We then assess the evidence of companionship ( $\S 3.1)$, measure spectral types $(\S 3.2)$, and estimate the physical properties of these companions ( $\S 3.3$ ). Finally, we discuss these newly discovered $\mathrm{T}$ dwarfs in the context of previously known brown dwarfs $(\S 4)$.

\section{OBSERVATIONS}

\subsection{Spitzer Mid-IR Imaging}

As a part of the Guaranteed Time Observations of the IRAC instrument team, we obtained images at 3.6, 4.5, 5.8, and $8.0 \mu \mathrm{m}$ with IRAC on board the Spitzer Space Telescope of 73 nearby young stars $(\mathrm{PID}=34)$ and 48 known extrasolar planetary systems $(P I D=48)$ between late 2003 and early 2005 . The plate scale and field of view of IRAC are $1.2^{\prime \prime}$ and $5.2^{\prime} \times 5.2^{\prime}$, respectively. The camera produces images with FWHM $=1.6^{\prime \prime}-1.9^{\prime \prime}$ from 3.6 to $8.0 \mu \mathrm{m}$ (Fazio et al. 2004). The observing strategy and image reduction procedures were the same as those described by Patten et al. (2006) for IRAC observations of M, L, and T dwarfs, except for the following modifications (Patten et al. 2007). The data were processed using the software suite IRACproc developed for this program to facilitate the combining of dithered or mapped

\footnotetext{
${ }^{11}$ In a paper submitted after this one, Mugrauer et al. (2006) reported an independent discovery of the companion to HD 3651.
}

IRAC data and the rejection of cosmic rays (Schuster et al. 2006). In order to obtain the best possible photometry of faint sources, we removed the light from each primary by fitting a scaled IRAC point-spread function in each band that was derived from IRAC observations of Vega, $\epsilon$ Eri, and $\epsilon$ Ind A (Marengo et al. 2006).

We measured photometry and astrometry of all point sources appearing in the reduced images of each star. We then identified candidate $\mathrm{T}$ dwarf companions by comparing these data to the colors of known T dwarfs (Patten et al. 2006), which are highly distinctive from those of other astronomical sources. We did not search for $\mathrm{M}$ and $\mathrm{L}$ dwarf companions because their IRAC colors do not differ significantly from those of stars at earlier types. We also considered only candidates that had magnitudes that were consistent with the absolute magnitudes of known $\mathrm{T}$ dwarfs when placed at the distance of the primary. These selection criteria were applied with a statistical technique based on the $k$-nearest neighbor ( $k$-NN; Fix \& Hodges 1951), which is a nonparametric classifier appropriate when a physical model is not available or is not reliable enough for fitting the data. We adapted this data mining technique, commonly used in pattern recognition and unsupervised machine learning, to the case of astronomical photometric data. By defining an appropriate metric in the color and magnitude multidimensional space, we determined a "score" for each object, based on its "distance" from the colors and magnitudes of known T dwarfs. A full description of our technique is presented in M. Marengo \& M. C. Sanchez (2007, in preparation). The two candidates with the best scores appeared in the images of HN Peg and HD 3651. Their positions in the IRAC colorcolor and color-magnitude diagrams are indicated in Figures 1 and 2, respectively, and their photometric measurements are listed in Table 1. No other objects had scores that were comparable to those for these two candidates. Follow-up observations of these candidates are described in $\S 2.2$.

During a survey for debris disks with the Multiband Imaging Photometer for Spitzer, Bryden et al. (2006) obtained $24 \mu \mathrm{m}$ images of HN Peg. These data encompass the candidate companion to this star that we have found with IRAC. According to analysis that was kindly performed by G. Bryden, the candidate is not detected in those $24 \mu \mathrm{m}$ data. Extrapolating from the IRAC data with a Rayleigh-Jeans distribution, the expected $24 \mu \mathrm{m}$ flux 


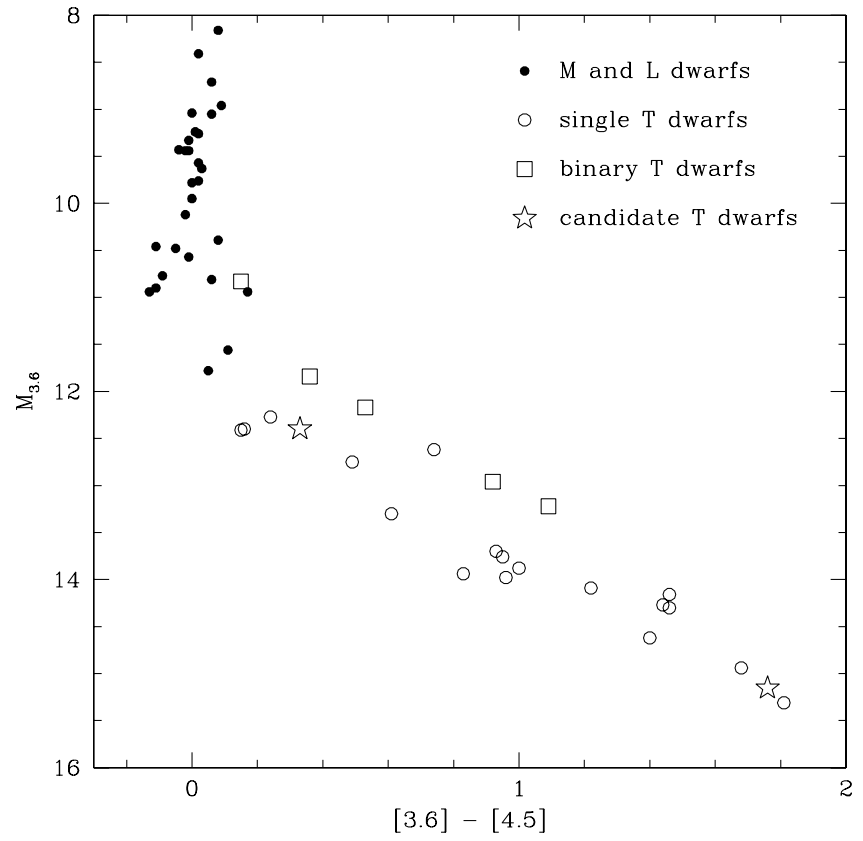

FIG. 2.-IRAC color-magnitude diagram for cool field dwarfs that have measured distances (open and filled circles and squares; Patten et al. 2006) and two candidate companions of HN Peg (left star) and HD 3651 (right star). The absolute magnitudes of the candidates have been computed by adopting the distances of the primaries. The magnitudes and colors of these candidate companions are indicative of $\mathrm{T}$ dwarfs. Unlike known $\mathrm{T}$ dwarf binaries (Burgasser et al. 2007 and references therein), HN Peg and HD 3651 are near the lower envelope of the $\mathrm{T}$ dwarf sequence, indicating that they are probably not equal-component binaries.

from the candidate is $\sim 0.1 \mathrm{mJy}$, while the noise in the $24 \mu \mathrm{m}$ image is about twice this value. These relatively shallow observations were designed to detect an excess above the stellar photosphere of HN Peg rather than a faint companion.

\subsection{IRTF Near-IR Imaging and Spectroscopy}

We obtained near-IR spectra of the candidate T dwarf companions to HN Peg and HD 3651 with the spectrometer SpeX (Rayner et al. 2003) at the NASA Infrared Telescope Facility (IRTF) on the nights of 2006 June 15 and 30. The instrument was operated in the prism mode with a $0.8^{\prime \prime}$ slit, producing a wavelength coverage of $0.8-2.5 \mu \mathrm{m}$ and a resolution of $R \sim 100$. For each candidate, we obtained 20 exposures distributed between two positions along the slit. The individual exposure times were 1.5 minutes, resulting in a total exposure time of 30 minutes for each object. The slit was aligned to the parallactic angle for all observations. The spectra were reduced with the Spextool package (Cushing et al. 2004) and corrected for telluric absorption (Vacca et al. 2003).

We also used the slit-viewing camera on SpeX to measure near-IR photometry for the candidate companions on the night of 2006 July 2 . The camera contained a $512 \times 512$ Aladdin 2 InSb array and had a plate scale of $0.12^{\prime \prime}$ pixel $^{-1}$ (Rayner et al.

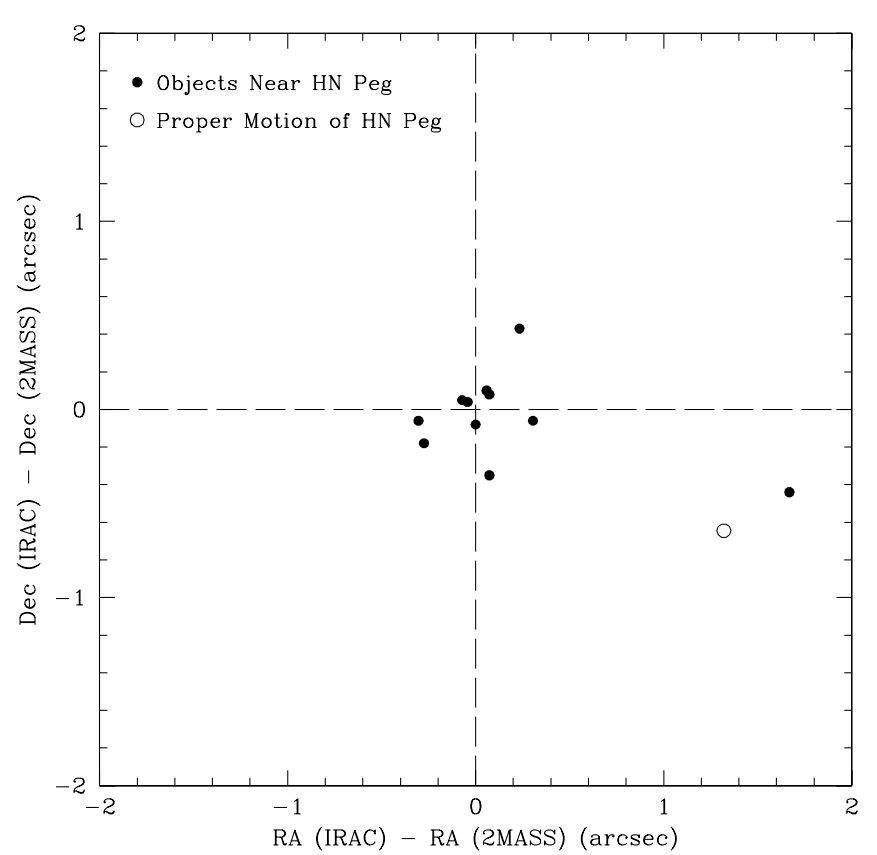

FIG. 3.-Differences in coordinates of sources near HN Peg between an $H$-band 2MASS image obtained in 1998 and a $4.5 \mu \mathrm{m}$ IRAC image obtained in 2004 (filled circles). The expected proper motion of HN Peg during this time interval is indicated (open circle; Perryman et al. 1997). Excluding the candidate $\mathrm{T}$ dwarf companion from Figs. 1 and 2 (rightmost filled circle), the standard deviations of the offsets are $\sigma_{\mathrm{RA}}=0.19^{\prime \prime}$ and $\sigma_{\mathrm{Dec}}=0.20^{\prime \prime}$, which represent the errors in these measurements. The candidate $\mathrm{T}$ dwarf exhibits a proper motion that is nonzero at a level of $8.8 \sigma_{\mathrm{RA}}$ and $2.2 \sigma_{\mathrm{Dec}}$ and is consistent with that of HN Peg at a level of $1.8 \sigma_{\mathrm{RA}}$ and $1.0 \sigma_{\mathrm{Dec}}$, demonstrating that it is a companion.

2003). Imaging was performed through Mauna Kea Observatory (MKO) $J, H$, and $K$ filters (Simons \& Tokunaga 2002; Tokunaga et al. 2002; Tokunaga \& Vacca 2005). In each filter, we obtained one $30 \mathrm{~s}$ exposure at each of nine dithered positions. For the $K$-band imaging of the candidate companion to HD 3651 , we performed this cycle twice. Images for a given candidate and filter were median combined to produce a flat-field image, which was then divided into the original exposures. Point sources in these images exhibited FWHM $=0.8^{\prime \prime}$. We extracted photometry for each candidate companion with the task phot under the IRAF package apphot using a radius of 8 pixels. These data were calibrated with images of standard stars for the MKO system (Leggett et al. 2006). The $J, H$, and $K$ measurements for the two candidate companions are presented in Table 1.

\section{ANALYSIS}

\subsection{Evidence of Binarity}

When a candidate companion is identified in direct imaging of a star in the solar neighborhood, the standard method of definitively assessing its status as a binary companion consists of checking whether it shares the same proper motion as the primary. To apply this method to the two candidate T dwarfs from our IRAC images

TABLE 1

Astrometry and Photometry for Companions

\begin{tabular}{|c|c|c|c|c|c|c|c|c|c|}
\hline Companion & $\begin{array}{c}\rho \\
(\operatorname{arcsec})\end{array}$ & $\begin{array}{l}\text { P.A. } \\
\text { (deg) }\end{array}$ & $J(\mathrm{MKO})$ & $H(\mathrm{MKO})$ & $K(\mathrm{MKO})$ & [3.6] & [4.5] & {$[5.8]$} & {$[8.0]$} \\
\hline HN Peg B & $43.2 \pm 0.4$ & $254.4 \pm 0.6$ & $15.86 \pm 0.03$ & $15.40 \pm 0.03$ & $15.12 \pm 0.03$ & $13.72 \pm 0.04$ & $13.39 \pm 0.02$ & $13.08 \pm 0.10$ & $12.58 \pm 0.11$ \\
\hline HD $3651 \mathrm{~B} \ldots \ldots .$. & $42.9 \pm 0.4$ & $290.1 \pm 0.6$ & $16.16 \pm 0.03$ & $16.68 \pm 0.04$ & $16.87 \pm 0.05$ & $15.38 \pm 0.04$ & $13.62 \pm 0.02$ & $14.04 \pm 0.12$ & $13.45 \pm 0.14$ \\
\hline
\end{tabular}




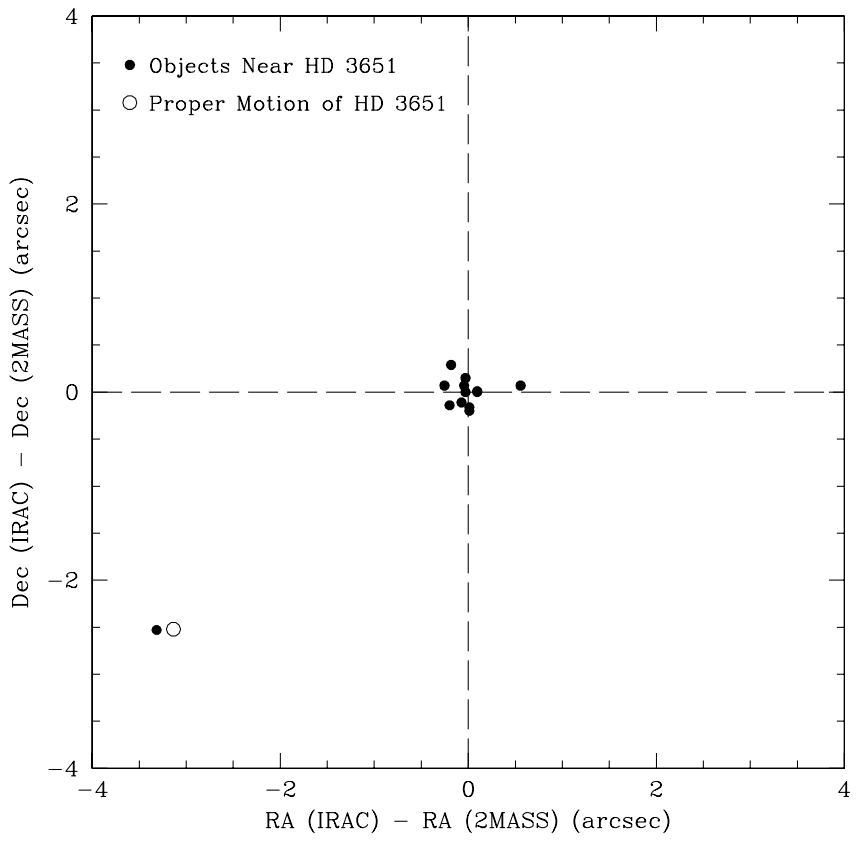

FIG. 4.-Differences in coordinates of sources near HD 3651 between a $J$-band 2MASS image obtained in 1997 and a $4.5 \mu \mathrm{m}$ IRAC image obtained in 2004 (filled circles). The expected proper motion of HD 3651 during this time interval is indicated (open circle; Perryman et al. 1997). Excluding the candidate $\mathrm{T}$ dwarf companion from Figs. 1 and 2 (leftmost filled circle), the standard deviations of the offsets are $\sigma_{\mathrm{RA}}=0.21^{\prime \prime}$ and $\sigma_{\mathrm{Dec}}=0.14^{\prime \prime}$, which represent the errors in these measurements. The candidate $\mathrm{T}$ dwarf exhibits a proper motion that is nonzero at a level of $16 \sigma_{\mathrm{RA}}$ and $18 \sigma_{\mathrm{Dec}}$ and is consistent with that of HD 3651 at a level of $0.8 \sigma_{\mathrm{RA}}$ and $0.1 \sigma_{\text {Dec }}$, demonstrating that it is a companion.

of HN Peg and HD 3651, we needed an image of each object obtained at a second epoch. The candidate companion to HN Peg was detected in each of the three 2MASS bands and appears in the 2MASS Point Source Catalog. The candidate near HD 3651 is not in the 2MASS Point Source Catalog, but through visual inspection of the images, we find that it was detected in $J$ and $H$.

To measure the proper motion of each candidate, we identified sources that appear in both the IRAC and the 2MASS images and measured their right ascensions and declinations in the $4.5 \mu \mathrm{m}$ image of IRAC and in the 2MASS band in which the candidate companion has the highest signal-to-noise ratio $(H$ for $\mathrm{HN}$ Peg and $J$ for HD 3651). The differences between the IRAC and 2MASS coordinates for these objects are plotted in Figures 3 and 4 . The relatively small number of stars in each diagram is a reflection of the requirement of a detection by $2 \mathrm{MASS}$; the numbers of sources detected by the IRAC images are much larger. To correct for a small offset between the coordinate systems of the IRAC and 2MASS images, we applied constant shifts to the right ascension and declination differences so that their average values were zero. In other words, we assume that the average proper motion of stars surrounding each star is zero. For comparison, we include in Figures 3 and 4 the expected displacements of HN Peg and HD 3651 during the time interval between the 2MASS and IRAC observations $(\Delta \tau=5.7$ and $6.8 \mathrm{yr})$ based on their known proper motions (Perryman et al. 1997). In each of the two diagrams, one source exhibits a motion that is significantly nonzero and that is consistent with the expected motion of the primary. These two objects are the candidate T dwarfs that we found with IRAC. Thus, these data demonstrate that both candidates have common proper motions with the primaries, which conclusively establishes their companionship. Hereafter in this paper, we refer to these new companions as HN Peg B and HD 3651B. ${ }^{12}$ The positions of HN Peg B and HD 3651B relative to their primaries are listed in Table 1 . The 2MASS and IRAC images of these systems are shown in Figures 5 and 6.

\subsection{Spectral Classification}

In $\S 2.1$, we identified HN Peg B and HD 3651B as possible $\mathrm{T}$ dwarfs based on their mid-IR colors and magnitudes. As shown in Figures 7 and 8, our near-IR spectra of both companions exhibit methane absorption, which is the defining spectral feature of T dwarfs (Kirkpatrick 2005). To measure the precise spectral type of each companion, we visually compared these spectra to the SpeX data for T dwarf standards that were presented by Burgasser et al. (2006b). In Figure 7, we find that the spectrum of HN Peg B agrees well with the average of the spectra of SDSS 1254-0122 (T2) and 2MASS 1209-1004 (T3). The match is clearly worse with either of these standards alone. Therefore, we assign a spectral type of $\mathrm{T} 2.5 \pm 0.5$ to $\mathrm{HN}$ Peg B.

Although the overall spectrum of HN Peg B is fit well with standard $\mathrm{T}$ dwarfs, the $\mathrm{K}$ I absorption lines at $1.25 \mu \mathrm{m}$ appear to be weaker in HN Peg B than in the standards. In comparison, these lines become weaker with lower gravity for late $M$ and early L dwarfs (McGovern et al. 2004; Kirkpatrick et al. 2006) but grow stronger with lower gravity at late T types (Knapp et al. 2004). Based on the apparent youth of its primary ( $\S 3.3 .1)$, HN Peg B should have a lower surface gravity than the standard $\mathrm{T}$ dwarfs

12 The planetary companion to HD 3651 from Fischer et al. (2003) is designated with a lowercase " $b$ " based on the naming convention for planets from radial velocity surveys.

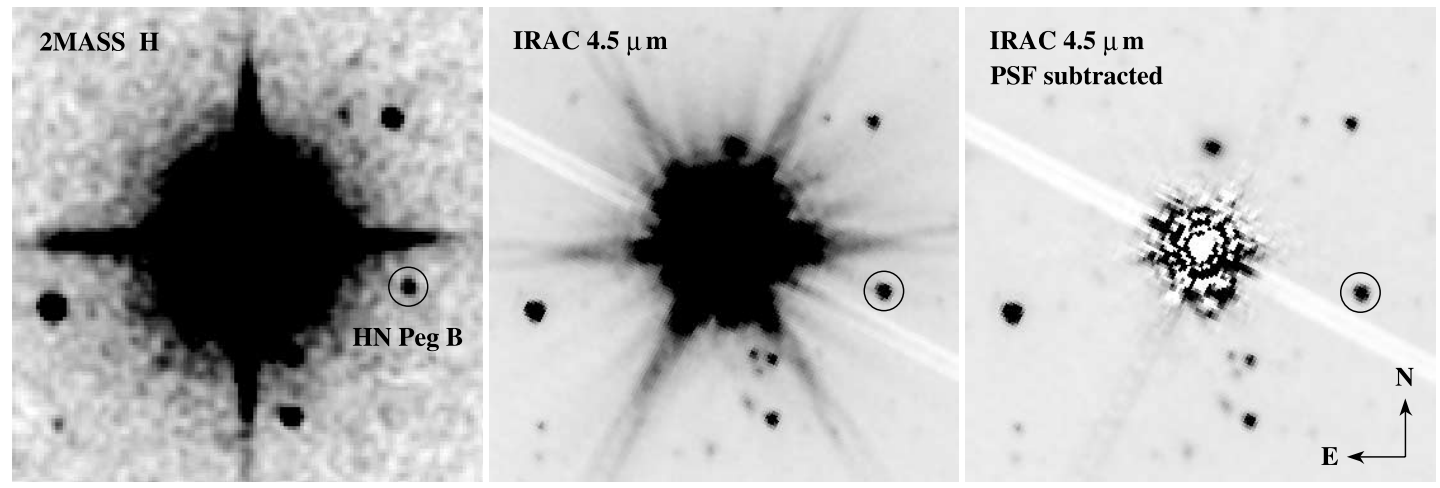

FIg. 5.-2MASS and IRAC images of HN Peg A and B. The size of each image is $2^{\prime} \times 2^{\prime}$. 


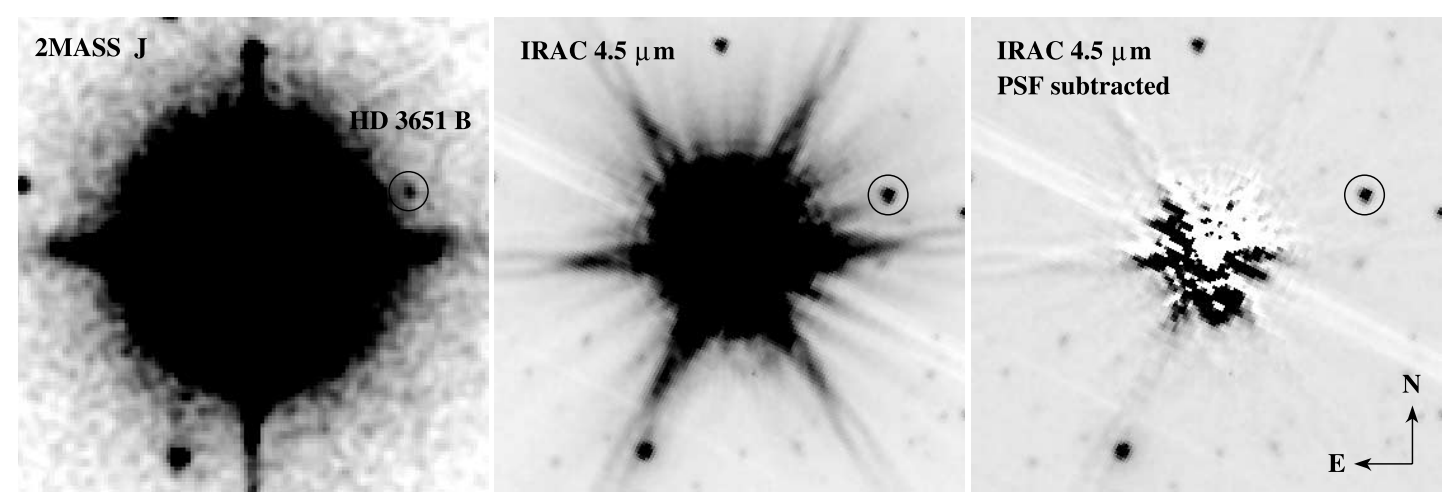

FIG. 6. - 2MASS and IRAC images of HD $3651 \mathrm{~A}$ and $\mathrm{B}$. The size of each image is $2^{\prime} \times 2^{\prime}$.

in Figure 7. Thus, these data for HN Peg B seem to indicate that the gravity dependence of the $\mathrm{K}$ I lines is the same in early $\mathrm{T}$ dwarfs as it is in $\mathrm{M}$ and $\mathrm{L}$ dwarfs. However, because the spectral resolution of our data is too low for reliable measurements of these lines, a spectrum at higher resolution is needed to explore this issue definitively.
In Figure 8, we compare HD 3651B to 2MASS $0727+1710$ (T7) and 2MASS 0415-0935 (T8) and the average of the two standards. The differences between HD 3651B and each of these three spectra are subtle, but close examination of each comparison indicates that the average of $\mathrm{T} 7$ and $\mathrm{T} 8$ provides the best match to HD 3651. For instance, the width of the continuum at $1.2-1.3 \mu \mathrm{m}$,

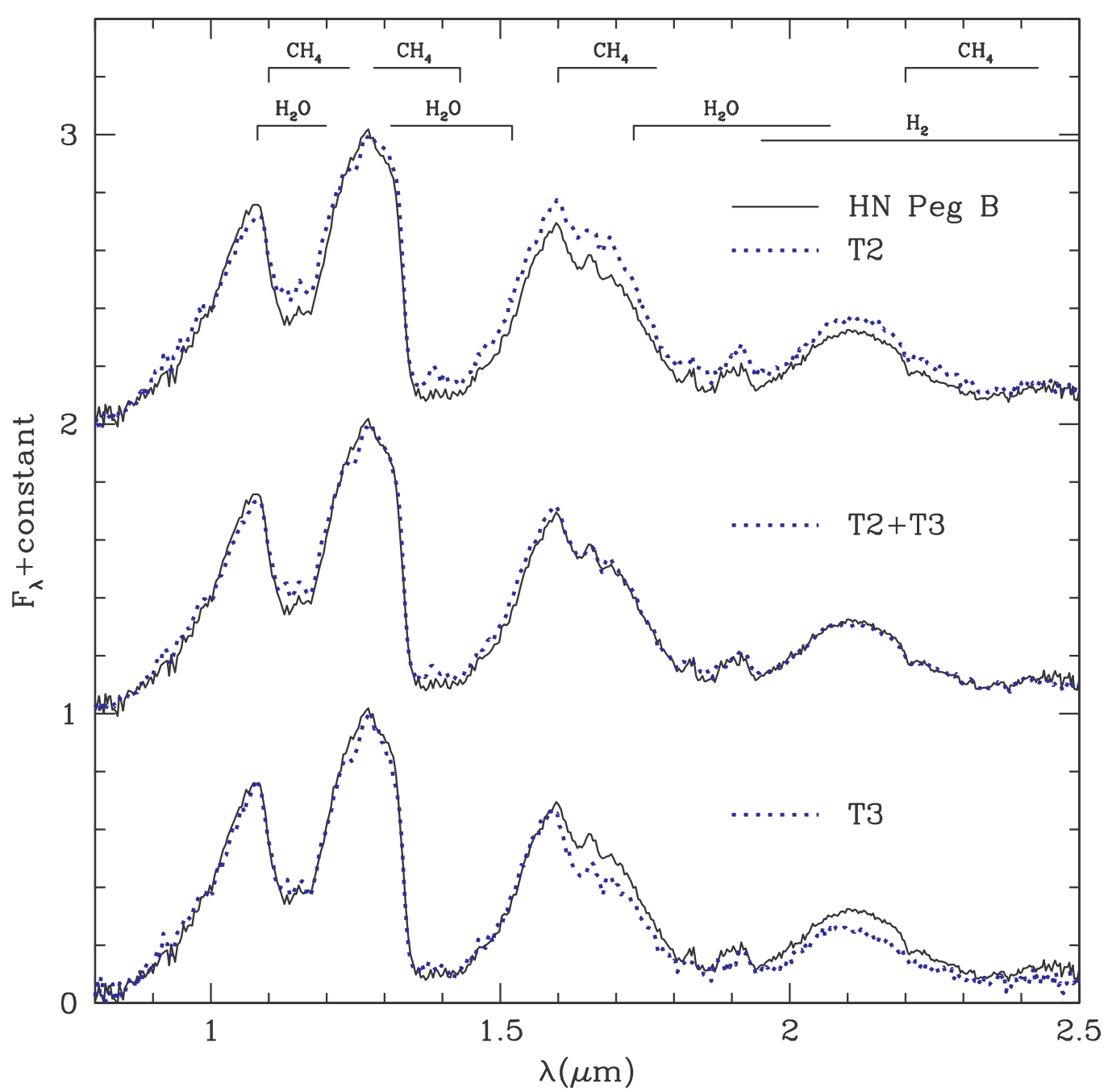

Fig. 7.-Near-IR spectrum of HN Peg B (solid line) compared to data for the standard T dwarfs SDSS 1254-0122 (T2) and 2MASS 1209-1004 (T3) from Burgasser et al. (2006b, dotted lines). The average of these standard spectra provides the best match to the spectrum of HN Peg B. The spectra have a resolution of $R=100$ and are normalized at $1.27 \mu \mathrm{m}$. 


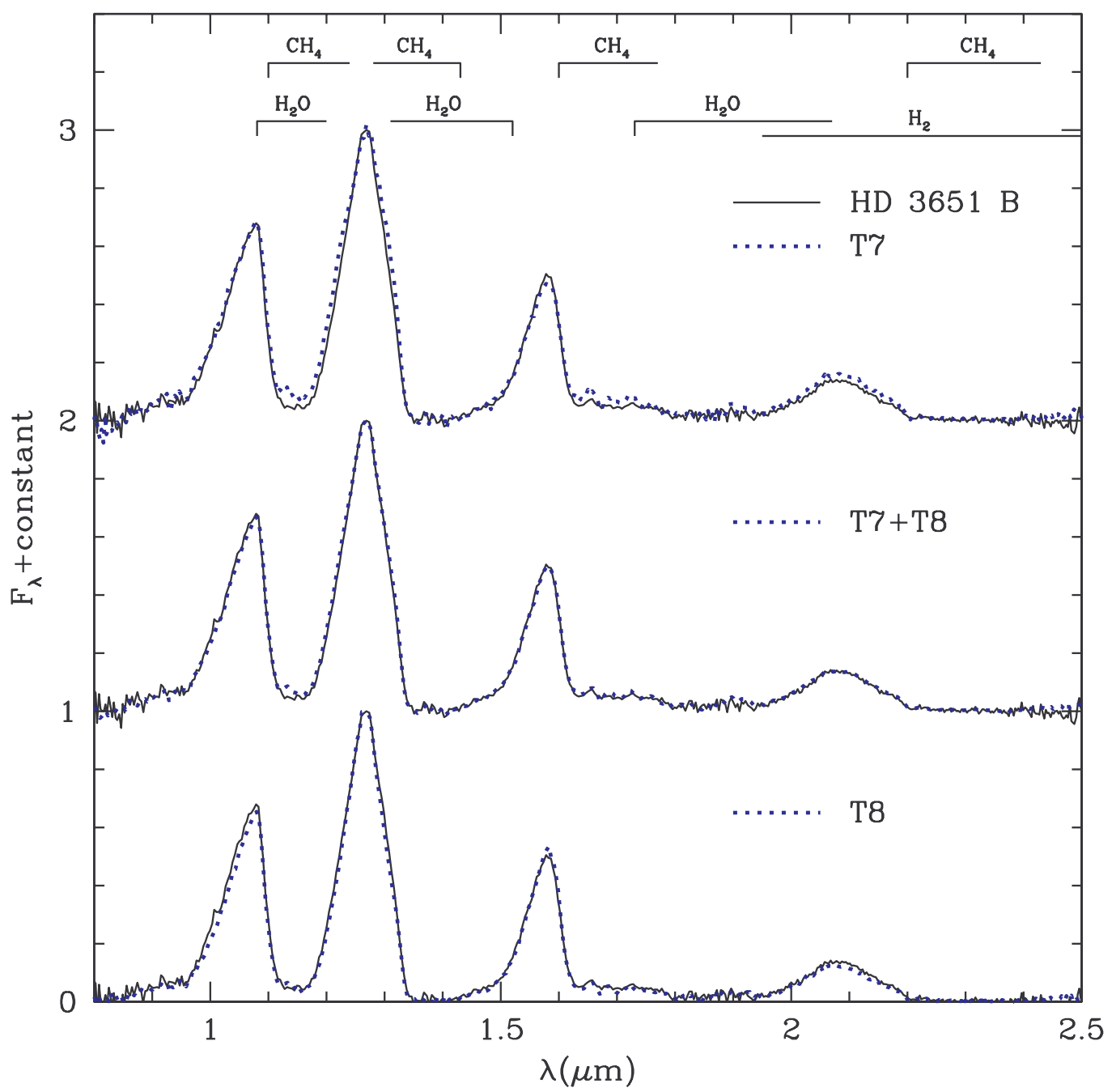

FIG. 8. - Near-IR spectrum of HD 3651 B (solid line) compared to data for the standard T dwarfs 2MASS 0727+1710 (T7) and 2MASS $0415-0935$ (T8) from Burgasser et al. (2006b, dotted lines). The average of these standard spectra provides the best match to the spectrum of HD $3651 \mathrm{~B}$. The spectra have a resolution of $R=100$ and are normalized at $1.27 \mu \mathrm{m}$.

the depth of the absorption at $1.6-1.8 \mu \mathrm{m}$, and the level of the $K$-band flux are all best fit by the average of $\mathrm{T} 7$ and $\mathrm{T} 8$. Therefore, we measure a spectral type of T7.5 \pm 0.5 for HD $3651 \mathrm{~B}$.

\subsection{Physical Properties \\ 3.3.1. Age and Distance}

Now that HN Peg B and HD 3651B have been confirmed as companion $\mathrm{T}$ dwarfs, we examine their physical properties. Because these $\mathrm{T}$ dwarfs are companions, we can assign to them the ages and distances of their primaries, which are bright, wellstudied stars in the solar neighborhood. As a result, the ages and distances of these T dwarfs, and hence the masses, radii, and temperatures, are better constrained than those of isolated $\mathrm{T}$ dwarfs. We adopt the Hipparcos trigonometric distances of $18.4 \pm 0.3$ and $11.1 \pm 0.1 \mathrm{pc}$ for HN Peg A and HD $3651 \mathrm{~A}$, respectively (Perryman et al. 1997). HN Peg A appears to be a young solar analog based on its activity, X-ray emission, rotation, Li abundance, and kinematics (Gaidos 1998). A comparison of the $\mathrm{Li}$ measurements for HN Peg A (Chen et al. 2001) to Li data for solar-type members of the Pleiades and Hyades open clusters and the Ursa Major moving group (Soderblom et al. 1990, 1993a, 1993b) indicates that HN Peg A is probably older than the Pleiades ( $\tau=100-125$ Myr; Meynet et al. 1993; Stauffer et al. 1998) and younger than Ursa Major ( $\tau=400-600 \mathrm{Myr}$; King et al. 2003) and the Hyades $(\tau=575-675$ Myr; Perryman et al. 1998). A comparison of the rotational properties of HN Peg A ( $P=4-5$ days, $v \sin i \sim 10 \mathrm{~km} \mathrm{~s}^{-1}$, e.g., König et al. 2006; Nordström et al. 2004) and these open clusters (Radick et al. 1987; Soderblom et al. 1993c; Soderblom \& Mayor 1993) produces the same result. The chromospheric activity of HN Peg A suggests an age of 0.3-0.4 Gyr (Gaidos 1998; Rocha-Pinto et al. 2004). A debris disk was recently detected around HN Peg A at 30 and $70 \mu \mathrm{m}$ by the Spitzer Space Telescope (Bryden et al. 2006; Beichman et al. 2006), which tends to further support the youth of this star. Meanwhile, HD 3651 is probably older than the Sun considering its fairly long rotational period ( $P=48$ days; Noyes et al. 1984). Wright et al. (2004) estimated an age of 5.9 Gyr from its chromospheric activity, and Valenti \& Fischer (2005) reported an isochronal age of $8.2_{-5.2}^{+4.3}$ Gyr. Based on these age constraints, we adopt ages of $0.3 \pm 0.2$ and $7 \pm 3$ Gyr for the HN Peg and HD 3651 systems, respectively.

\subsubsection{Luminosity}

To measure the bolometric luminosities of HN Peg B and HD $3651 \mathrm{~B}$, we first used the $J H K$ photometry to flux calibrate the $0.8-2.5 \mu \mathrm{m}$ spectra. For each object, we constructed a spectral energy distribution that consisted of these calibrated spectra, the 

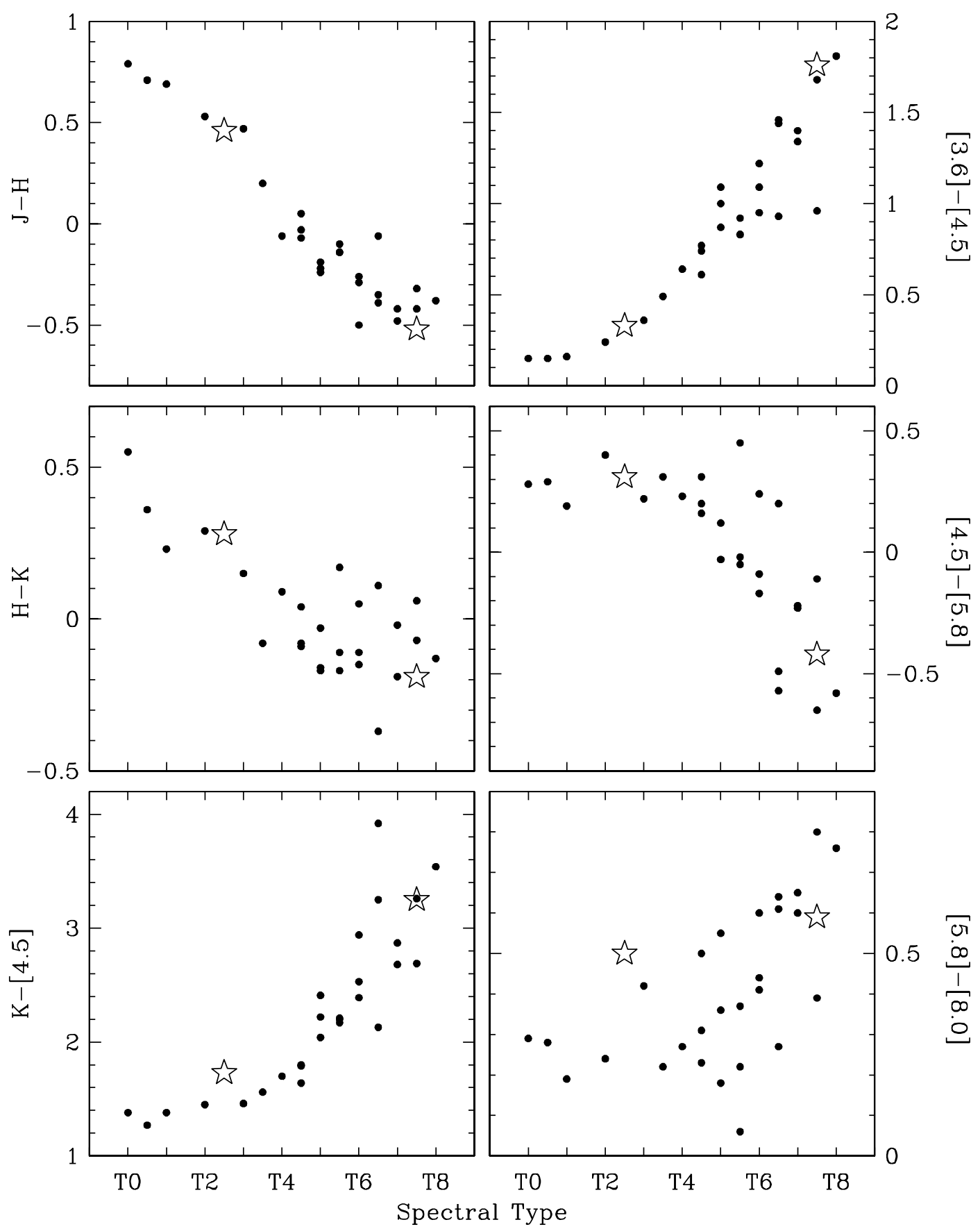

Fig. 9.- Infrared colors vs. spectral type for previously known T dwarfs (Patten et al. 2006, filled circles) and the new T dwarf companions HN Peg B (left stars) and HD 3651 B (right stars).

IRAC photometry $(3.2-9.2 \mu \mathrm{m})$, a linear interpolation of the fluxes between 2.5 and $3.2 \mu \mathrm{m}$, and a Rayleigh-Jeans distribution longward of $9.2 \mu \mathrm{m}$. By summing the flux in each distribution and correcting for distance, we measured $\log L / L_{\odot}=-4.77 \pm 0.03$ and $-5.60 \pm 0.05$ for HN Peg B and HD 3651B, respectively.

Our luminosity measurement for HN Peg B is greater than the value produced by combining its $K$ magnitude with the bolometric corrections $\left(\mathrm{BC}_{K}\right)$ that have been previously measured for T2-T3 dwarfs (Golimowski et al. 2004). For instance, the bolometric luminosity for the T2 dwarf SDSS 1254-0122 from Cushing et al. (2006) implies $\mathrm{BC}_{K}=3.04$. If we combine this value of $\mathrm{BC}_{K}$ with our $K$ measurement for $\mathrm{HN}$ Peg B and an absolute bolometric magnitude for the Sun of $M_{\mathrm{bol} \odot}=4.75$, we arrive at $\log L / L_{\odot}=-4.83$. To explore the source of the differ- ence in these two luminosity estimates, we compare in Figure 9 the near- and mid-IR colors of HN Peg B to colors of T dwarfs compiled by Patten et al. (2006). The 2MASS measurements from Patten et al. (2006) have been transformed to the MKO system (Stephens \& Leggett 2004). In the diagrams of colors versus spectral type in Figure 9, HN Peg B is similar to other T dwarfs near its spectral type in terms of $J-H, H-K$, [3.6]-[4.5], and [4.5]-[5.8], but it is redder by $\sim 0.3$ and $\sim 0.15$ mag in $K-[4.5]$ and [5.8]-[8.0], respectively. The latter difference is not significant because of the large photometric error in [8.0]. However, HN Peg B clearly has redder JHK-IRAC colors relative to typical field dwarfs, which explains why the luminosity derived from $K$ and $\mathrm{BC}_{K}$ was lower than the value measured from the photometry and spectra. In other words, if 

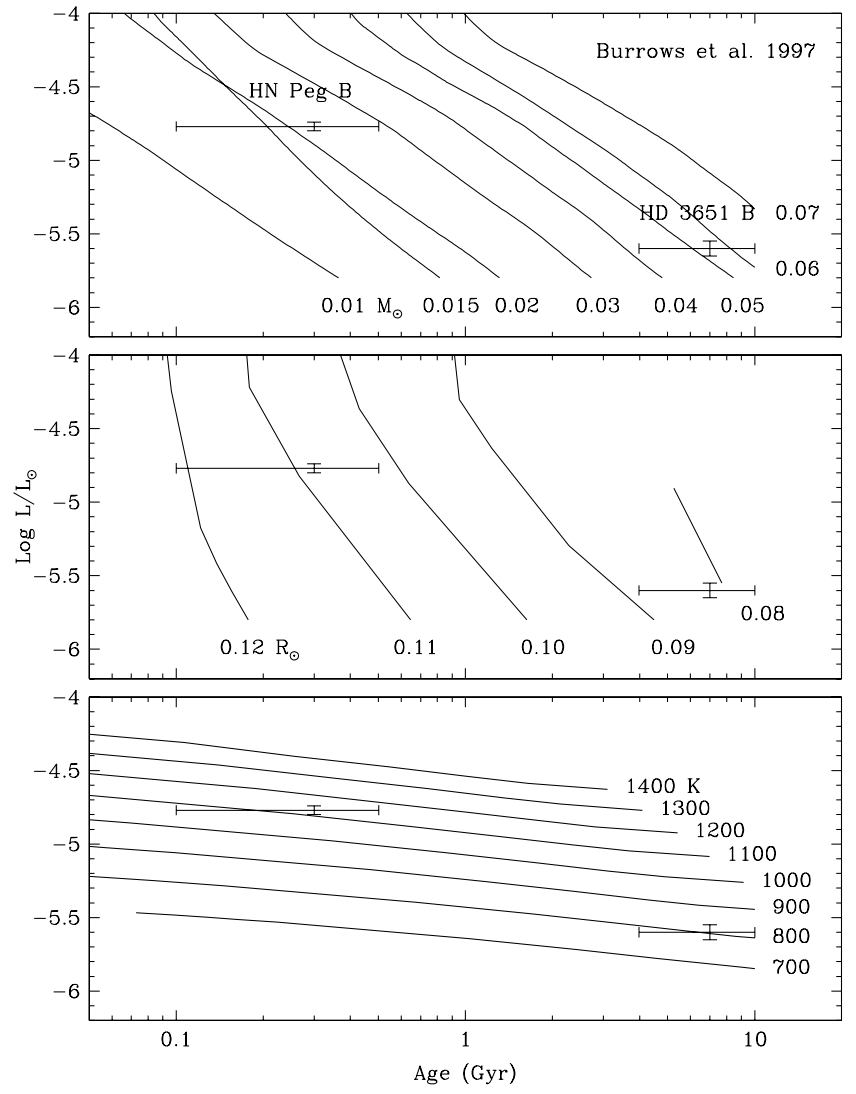

Fig. 10.-Luminosities of HN Peg B and HD 3651 B compared to the luminosities as a function of age predicted by the theoretical evolutionary models of Burrows et al. (1997) (lines) for constant values of mass, radius, and temperature (top to bottom).

we reduced the IRAC fluxes of HN Peg B by $\sim 30 \%$ so that its $J H K$-IRAC colors were consistent with those of other T2-T3 dwarfs like SDSS 1254-0122, then our luminosity measurement would agree with the one based on $K$ and $\mathrm{BC}_{K}$.

Our luminosity measurement for HD $3651 \mathrm{~B}$ agrees well with the value produced by combining its $K$ magnitude with $\mathrm{BC}_{K}$ for T7-T8 dwarfs from Golimowski et al. (2004). In addition, the near- and mid-IR colors of HD $3651 \mathrm{~B}$ are consistent with those of the other T dwarfs shown in Figure 9.

\subsubsection{Mass, Radius, and Temperature}

We use theoretical evolutionary models to convert our luminosity and age estimates for HN Peg B and HD 3651B to masses, effective temperatures, and radii. We perform this conversion by plotting these companions on diagrams of luminosity versus age and comparing their positions to lines of constant mass, temperature, and radius that are predicted by evolutionary models. These diagrams are presented in Figures 10 and 11 with the models of Burrows et al. (1997) and Baraffe et al. (2003) respectively, which imply nearly identical physical properties. The models produce $M=0.021 \pm 0.009 M_{\odot}, R=0.108_{-0.006}^{+0.014} R_{\odot}$, and $T_{\text {eff }}=$ $1130 \pm 70 \mathrm{~K}$ for HN Peg B and $M=0.051 \pm 0.014 M_{\odot}, R=$ $0.082 \pm 0.006 R_{\odot}$, and $T_{\text {eff }}=810 \pm 50 \mathrm{~K}$ for HD $3651 \mathrm{~B}$. The spectral types and physical properties of HN Peg B and HD 3651B are summarized in Table 2.

\section{DISCUSSION}

We have presented the discovery of two T dwarf companions in the solar neighborhood, HN Peg B (T2.5) and HD 3651B
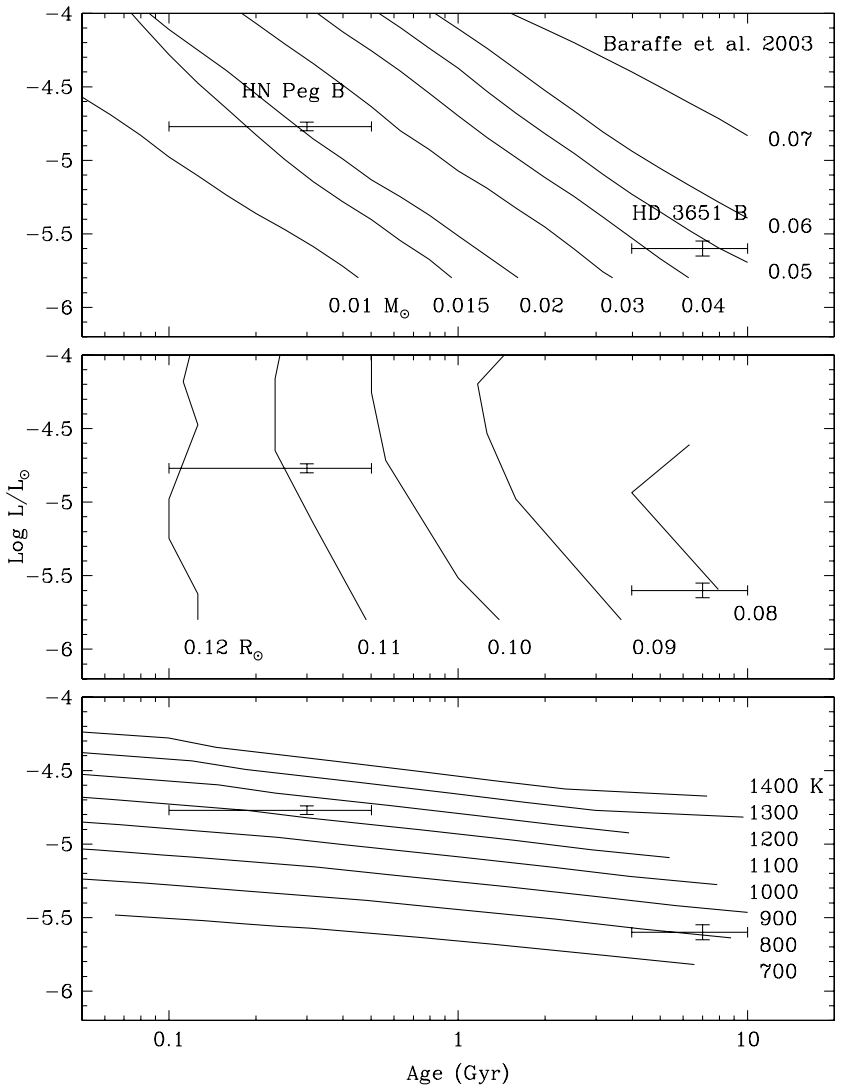

FIG. 11.-Same as Fig. 10, but with the models of Baraffe et al. (2003).

(T7.5). These T dwarfs are the sixth and seventh known T dwarf companions to stars and are the first $\mathrm{T}$ dwarfs discovered with the Spitzer Space Telescope. In this section, we describe additional notable aspects of these new brown dwarfs.

HN Peg A has been the target of high-precision radial velocity monitoring (Cumming et al. 1999; Fischer \& Valenti 2005; König et al. 2006) and near-IR coronographic imaging (McCarthy \& Zuckerman 2004), but no companions have been found through those observations. HN Peg B was bright enough to be detected by the imaging of McCarthy \& Zuckerman (2004), but it was outside of their field of view $\left(r<15^{\prime \prime}\right)$. This companion was also beyond the field of view of unpublished images of HN Peg obtained with the Near-Infrared Camera and Multi-Object Spectrometer on board the Hubble Space Telescope during General Observer program 10176. Various data from the literature indicate that HN Peg A has an age of 0.1-0.5 Gyr (§ 3.3.1). If the T dwarf S Ori 70 (Zapatero Osorio et al. 2002) is a field dwarf rather than a member of the young cluster $\sigma$ Ori (Burgasser et al. 2004), then HN Peg B may be the youngest known T dwarf. HN Peg B closely resembles the recently discovered late L companion to HD 203030 in terms of age and mass (Metchev \& Hillenbrand 2006).

Several properties of HN Peg B do not behave in the manner predicted for young, low-gravity T dwarfs. Although the colors within the $J H K$ bands and within the IRAC bands are normal, all colors containing one of the former and one of the latter are redder than those of typical T dwarfs by $\sim 0.3$ mag ( $\S 3.3 .2$ ). In contrast, low gravity should cause these colors to become bluer, not redder, according to the theoretical spectra of Burrows et al. (2006). Based on its position in color-magnitude diagrams, HN Peg B is probably not an equal-component binary, but it could be a binary with two 
TABLE 2

Properties of Companions

\begin{tabular}{|c|c|c|c|c|c|c|c|c|}
\hline Companion & $\begin{array}{l}\text { Distance }^{\mathrm{a}} \\
\quad(\mathrm{pc})\end{array}$ & $\begin{array}{c}\rho^{\mathrm{b}} \\
(\mathrm{AU})\end{array}$ & $\begin{array}{l}\text { Spectral } \\
\text { Type }\end{array}$ & $\begin{array}{l}\text { Age } \\
\text { (Gyr) }\end{array}$ & $\log L / L_{\odot}$ & $M / M_{\odot}^{\mathrm{c}}$ & $R / R_{\odot}{ }^{\mathrm{c}}$ & $\begin{array}{l}T_{\text {eff }}^{\mathrm{c}} \\
(\mathrm{K})\end{array}$ \\
\hline 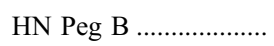 & $18.4 \pm 0.3$ & $795 \pm 15$ & $\mathrm{~T} 2.5 \pm 0.5$ & $0.3 \pm 0.2$ & $-4.77 \pm 0.03$ & $0.021 \pm 0.009$ & $0.108_{-0.006}^{+0.014}$ & $1130 \pm 70$ \\
\hline
\end{tabular}

${ }^{\text {a }}$ Distance of primary (Perryman et al. 1997).

${ }^{\mathrm{b}}$ Projected physical separation from primary.

${ }^{c}$ Estimated from the age, $\log L / L_{\odot}$, and the models of Burrows et al. (1997) and Baraffe et al. (2003) in Figs. 10 and 11.

unequal components. ${ }^{13}$ Because later $\mathrm{T}$ dwarfs emit proportionately more flux in the mid-IR bands than at shorter wavelengths, an unresolved system of this kind (e.g., T1+T5) might be responsible for these anomalous colors. Another possible explanation is photometric variability between the dates of the IRAC and JHK observations (Burgasser et al. 2002; Enoch et al. 2003).

Our estimates of the stellar parameters of HN Peg B also exhibit peculiarities. When we compare the luminosity and age of $\mathrm{HN}$ Peg B to the predictions of theoretical evolutionary models, we derive an effective temperature of $1130 \pm 70 \mathrm{~K}$, which is lower than the values previously measured for $\mathrm{T}$ dwarfs at $\mathrm{T} 2-\mathrm{T} 3$ $\left(T_{\text {eff }} \sim 1400 \mathrm{~K}\right.$, Golimowski et al. 2004). In contrast, the spectral modeling of late $\mathrm{T}$ dwarfs by Burgasser et al. (2006b) found that temperature should increase rather than decrease with lower gravity at a given spectral type. If this prediction is combined with the models shown in Figures 10 and 11, then a young T dwarf should be much brighter than an older one at the same spectral type. Instead, we find that HN Peg B is at the faint end of T0-T4 dwarfs (Golimowski et al. 2004) and is at least 1 mag fainter in bolometric luminosity than expected for its assumed age. One possible explanation for these discrepancies is that the HN Peg system is much older than various data for the primary seem to indicate (§ 3.3.1). However, Metchev \& Hillenbrand (2006) have recently reported the same anomalous behavior for a late L companion near another star that appears to be young, HD 203030, and it seems unlikely that the age estimates for both stars have such large errors. Instead, the presence of this phenomenon in both HN Peg B and HD 203030B strongly indicates that the temperature of the $\mathrm{L} / \mathrm{T}$ transition is dependent on surface gravity and in a manner that has not been previously predicted.

When combined with the evolutionary models, the luminosity and age of HN Peg B translate to a mass $0.021 \pm 0.009 M_{\odot}$, making it one of the least massive known T dwarfs. In addition, like HD 203030B $\left(M=0.023 M_{\odot}\right.$; Metchev \& Hillenbrand 2006), HN Peg B is among the least massive known brown dwarf

\footnotetext{
13 One of the T dwarfs that has been unresolved in previous imaging, 2MASS 0559-1404 (Burgasser et al. 2003), is overluminous in Fig. 2, which suggests that it could be a binary. This brown dwarf was identified as a possible binary because of its bright $J$ magnitude relative to late $L$ dwarfs (Dahn et al. 2002), but more recent 1-5 $\mu \mathrm{m}$ measurements indicated that it might not be significantly overluminous compared to other T dwarfs (Golimowski et al. 2004; Vrba et al. 2004).
}

companions and is a more evolved counterpart to the widely separated companions DH Tau B ( $\tau \sim 1$ Myr; Itoh et al. 2005), CHXR 73B ( $\tau \sim 2$ Myr; Luhman et al. 2006), and AB Pic B ( $\tau \sim 30 \mathrm{Myr}$; Chauvin et al. 2005b), which have masses of 0.01$0.02 M_{\odot}$ (Luhman et al. 2006).

For HD 3651, radial velocity monitoring has uncovered a sub-Saturn-mass planet at 0.3 AU (Fischer et al. 2003; Fischer \& Valenti 2005), while no companions have been identified in adaptive optics images (Carson et al. 2005). The latter observations did not detect HD 3651B because their field of view was too small $\left(r<15^{\prime \prime}\right)$. Takeda et al. (2007) suggested that the high eccentricities of planets in old, single-planet systems like HD 3651 might be caused by secular perturbations from distant low-mass companions, and indeed we have found such a companion in the form of HD 3651B. This T dwarf is the first resolved substellar companion that has been discovered in one of the extrasolar planetary systems identified in radial velocity surveys. Like $\mathrm{HN}$ Peg B, HD 3651B is probably not an equal-magnitude binary brown dwarf according to its position in the color-magnitude diagram in Figure 2. HD 3651B is a virtual twin to another T dwarf companion, Gl 570D, in terms of spectral type, luminosity, mass, temperature, and radius (Burgasser et al. 2000; Geballe et al. 2001). As with Gl 570D, these measurements have relatively high accuracy because of the companionship of HD 3651B to a nearby, well-studied star, making it a valuable brown dwarf for calibrating methods of characterizing the physical properties of late T dwarfs (Burgasser et al. 2006a).

We thank Eric Mamajek for discussions regarding the ages of HN Peg and HD 3651 and Adam Burgasser for providing his spectra of T dwarf standards. We are also grateful to Geoff Bryden for checking his $24 \mu \mathrm{m}$ images of HN Peg for a detection of the companion. K. L. was supported by grant NAG5-11627 from the NASA Long-Term Space Astrophysics program. This work is based on observations made with the Spitzer Space Telescope, which is operated by the Jet Propulsion Laboratory (JPL) at the California Institute of Technology under NASA contract 1407. Support for the IRAC instrument was provided by NASA through contract 960541 issued by JPL.
Baraffe, I., Chabrier, G., Barman, T. S., Allard, F., \& Hauschildt, P. H. 2003 , A\&A, 402, 701

Beichman, C. A., et al. 2006, ApJ, 639, 1166

Biller, B. A., Kasper, M., Close, L. M., Brandner, W., \& Kellner, S. 2006, ApJ, 641, L141

Bryden, G., et al. 2006, ApJ, 636, 1098

Burgasser, A. J., Burrows, A., \& Kirkpatrick, J. D. 2006a, ApJ, 639, 1095

Burgasser, A. J., Geballe, T. R., Leggett, S. K., Kirkpatrick, J. D., \& Golimowski, D. A. 2006b, ApJ, 637, 1067

Burgasser, A. J., Kirkpatrick, J. D., \& Lowrance, P. J. 2005, AJ, 129, 2849

\section{REFERENCES}

Burgasser, A. J., Kirkpatrick, J. D., McGovern, M. R., McLean, I. S., Prato, L., \& Reid, I. N. 2004, ApJ, 604, 827

Burgasser, A. J., Kirkpatrick, J. D., Reid, I. N., Brown, M. E., Miskey, C. L., \& Gizis, J. E. 2003, ApJ, 586, 512

Burgasser, A. J., Reid, I. N., Siegler, N., Close, L., Allen, P., Lowrance, P., \& Gizis, J. 2007, in Protostars and Planets V, ed. B. Reipurth, D. Jewitt, \& K. Keilin (Tucson: Univ. Arizona Press), in press

Burgasser, A. J., et al. 2000, ApJ, 531, L57

. 2002, ApJ, 571, L151

Burrows, A., Sudarsky, D., \& Hubeny, I. 2006, ApJ, 640, 1063 
Burrows, A., et al. 1997, ApJ, 491, 856

Carson, J. C., Eikenberry, S. S., Brandl, B. R., Wilson, J. C., \& Hayward, T. L. 2005, AJ, 130, 1212

Chauvin, G., et al. 2003, A\&A, 404, 157

. 2004, A\&A, 425, L29

- 2005a, A\&A, 438, L25

- 2005b, A\&A, 438, L29

. 2006, A\&A, 456, 1165

Chen, Y. Q., Nissen, P. E., Benoni, T., \& Zhao, G. 2001, A\&A, 371, 943

Cumming, A., Marcy, G. W., \& Butler, R. P. 1999, ApJ, 526, 890

Cushing, M. C., Vacca, W. D., \& Rayner, J. T. 2004, PASP, 116, 362

Cushing, M. C., et al. 2006, ApJ, 648, 614

Dahn, C. C., et al. 2002, AJ, 124, 1170

Els, S. G., Sterzik, M. F., Marchis, F., Pantin, E., Endl, M., \& Kürster, M. 2001, A\&A, 370, L1

Enoch, M. L., Brown, M. E., \& Burgasser, A. J. 2003, AJ, 126, 1006

Fazio, G. G., et al. 2004, ApJS, 154, 10

Fischer, D. A., Butler, R. P., Marcy, G. W., Vogt, S. S., \& Henry, G. W. 2003, ApJ, 590, 1081

Fischer, D. A., \& Valenti, J. 2005, ApJ, 622, 1102

Fix, E., \& Hodges, J. L. 1951, Technical Report 21-49-004 4 (Randolph Field: USAF School of Aviation Medicine)

Gaidos, E. J. 1998, PASP, 110, 1259

Geballe, T. R., Saumon, D., Leggett, S. K., Knapp, G. R., Marley, M. S., \& Lodders, K. 2001, ApJ, 556, 373

Golimowski, D. A., et al. 2004, AJ, 127, 3516

Guenther, E. W., Neuhäuser, R., Huélamo, N., Brandner, W., \& Alves, J. 2001, A\&A, 365, 514

Itoh, Y., et al. 2005, ApJ, 620, 984

King, J. R., Villarreal, A. R., Soderblom, D. R., Gulliver, A. F., \& Adelman, S. J. 2003, AJ, 125, 1980

Kirkpatrick, J. D. 2005, ARA\&A, 43, 195

Kirkpatrick, J. D., et al. 2006, ApJ, 639, 1120

Knapp, G. R., et al. 2004, AJ, 127, 3553

König, B., Guenther, E. W., Esposito, M., \& Hatzes, A. 2006, MNRAS, 365, 1050

Lagrange, A.-M., Beust, H., Udry, S., Chauvin, G., \& Mayor, M. 2006, A\&A, 459,955

Leggett, S. K., et al. 2006, MNRAS, 373, 781

Lowrance, P. J., et al. 1999, ApJ, 512, L69

- 2000, ApJ, 541, 390

2005, AJ, 130,1845

Luhman, K. L., \& Jayawardhana, R. 2002, ApJ, 566, 1132

Luhman, K. L., et al. 2006, ApJ, 649, 894

Marengo, M., Megeath, S. T., Fazio, G. G., Stapelfeldt, K. R., Werner, M. W., \& Backman, D. E. 2006, ApJ, 647, 1437

McCarthy, C., \& Zuckerman, B. 2004, AJ, 127, 2871

McCaughrean, M. J., et al. 2004, A\&A, 413, 1029

McGovern, M. R., Kirkpatrick, J. D., McLean, I. S., Burgasser, A. J., Prato, L., \& Lowrance, P. J. 2004, ApJ, 600, 1020

Metchev, S. A., \& Hillenbrand, L. A. 2004, ApJ, 617, 1330 2006, ApJ, 651, 1166

Meynet, G., Mermilliod, J.-C., \& Maeder, A. 1993, A\&AS, 98, 477
Mugrauer, M., \& Neuhäuser, R. 2005, MNRAS, 361, L15

Mugrauer, M., Neuhäuser, R., Seifahrt, A., Mazeh, T., \& Guenther, E. 2005 , A\&A, 440, 1051

Mugrauer, M., Seifahrt, A., Neuhäuser, R., \& Mazeh, T. 2006, MNRAS, 373, L31

Nakajima, T., Oppenheimer, B. R., Kulkarni, S. R., Golimowski, D. A., Matthews, K., \& Durrance, S. T. 1995, Nature, 378, 463

Neuhäuser, R., et al. 2000a, A\&A, 354, L9

2000b, A\&A, 360, L39

Nordström, B., et al. 2004, A\&A, 418, 989

Noyes, R. W., Hartmann, L. W., Baliunas, S. L., Duncan, D. K., \& Vaughan, A. H. 1984, ApJ, 279, 763

Oppenheimer, B. R., Kulkarni, S. R., Nakajima, T., \& Matthews, K. 1995, Science, 270, 1478

Patience, J., et al. 2002, ApJ, 581, 654

Patten, B. M., et al. 2006, ApJ, 651, 502 2007, in Protostars and Planets V, ed. B. Reipurth, D. Jewitt, \& K.

Keilin (Tucson: Univ. Arizona Press), in press

Perryman, M. A. C., et al. 1997, A\&A, 323, L49 . 1998, A\&A, 331, 81

Radick, R. R., Thompson, D. T., Lockwood, G. W., Duncan, D. K., \& Baggett, W. E. 1987, ApJ, 321, 459

Rayner, J. T., et al. 2003, PASP, 115, 362

Rebolo, R., et al. 1998, Science, 282, 1309

Rocha-Pinto, H. J., Flynn, C., Scalo, J., Hänninen, J., Maciel, W. J., \& Hensler, G. 2004, A\&A, 423, 517

Scholz, R.-D., McCaughrean, M. J., Lodieu, N., \& Kuhlbrodt, B. 2003, A\&A, 398, L29

Schuster, M., Marengo, M., \& Patten, B. 2006, Proc. SPIE, 6270, 627020

Simons, D. A., \& Tokunaga, A. 2002, PASP, 114, 169

Soderblom, D. R., Jones, B. F., Balachandran, S., Stauffer, J. R., Duncan, D. K., Fedele, S. B., \& Hudon, J. D. 1993a, AJ, 106, 1059

Soderblom, D. R., \& Mayor, M. 1993, ApJ, 402, L5

Soderblom, D. R., Oey, M. S., Johnson, D. R. H., \& Stone, R. P. S. 1990, AJ, 99, 595

Soderblom, D. R., Pilachowski, C. A., Fedele, S. B., \& Jones, B. F. 1993b, AJ, 105,2299

Soderblom, D. R., Stauffer, J. R., Hudon, J. D., \& Jones, B. F. 1993c, ApJS, 85, 315

Stauffer, J. R., Schultz, G., \& Kirkpatrick, J. D. 1998, ApJ, 499, L199

Stephens, D. C., \& Leggett, S. K. 2004, PASP, 116, 9

Takeda, G., Ford, E. B., Sills, A., Rasio, F. A., Fischer, D. A., \& Valenti, J. A. 2007, ApJS, in press

Tokunaga, A. T., Simons, D. A., \& Vacca, W. D. 2002, PASP, 114, 180

Tokunaga, A. T., \& Vacca, W. D. 2005, PASP, 117, 421

Vacca, W. D., Cushing, M. C., \& Rayner, J. T. 2003, PASP, 115, 389

Valenti, J. A., \& Fischer, D. A. 2005, ApJS, 159, 141

Vrba, F. J., et al. 2004, AJ, 127, 2948

Werner, M. W., et al. 2004, ApJS, 154, 1

Wilson, J. C., Kirkpatrick, J. D., Gizis, J. E., Skrutskie, M. F., Monet, D. G., \& Houck, J. R. 2001, AJ, 122, 1989

Wright, J. T., Marcy, G. W., Butler, R. P., \& Vogt, S. S. 2004, ApJS, 152, 261

Zapatero Osorio, M. R., et al. 2002, ApJ, 578, 536 